\title{
Promoter region methylation and loss of protein expression of PTEN and significance in cervical cancer
}

\author{
QIUFENG QI ${ }^{1}$, YANG LING ${ }^{2}$, MING ZHU $^{1}$, LINYAN ZHOU ${ }^{3}$, MEIZHEN WAN $^{3}$, \\ YANQING BAO ${ }^{1}$ and YONGPING LIU $^{1,2}$ \\ ${ }^{1}$ Clinical Oncology Laboratory; Departments of ${ }^{2}$ Oncology and ${ }^{3}$ Pathology, \\ Changzhou Tumor Hospital Affiliated to Suzhou University, Changzhou, Jiangsu 213002, P.R. China
}

Received April 9, 2014; Accepted June 6, 2014

DOI: $10.3892 / b r .2014 .298$

\begin{abstract}
The genetic basis underlying cervical tumorigenesis and progression are largely unknown. Phosphatase and tensin homologue (PTEN) is a tumor suppressor gene, and genetic changes of PTEN occurs in various types of cancer suggesting that the inactivation of PTEN may play an important role in the pathogenesis of a variety of human malignancies. In the present study, 102 cervical cancer specimens were examined for the expression of the PTEN gene and promoter methylation using methylation-specific-polymerase chain reaction and immunohistochemistry. The PTEN gene mutation was also assessed using PCR single-strand conformational polymorphism. We examined the correlation between PTEN expression and its associated methylation status and the clinical characteristics of cervical cancer. The results showed that there was one case of an A to $\mathrm{G}$ point mutation on exon 9 of the PTEN gene in the cervical cancer tissues. This mutation caused the change of aspartic acid to glycine, and the rate of mutation was $1 \%$. The PTEN gene methylation rate of cervical cancer was $62 \%$ $(63 / 102)$ and the rate was associated with the International Federation of Gynecology and Obstetrics stage, cell differentiation, tumor size and lymph node metastasis $(\mathrm{P}<0.05)$. The positive rate of PTEN expression was $49 \%$ (50/102) in cervical carcinoma and the PTEN expression between stage I-II and III-IV [60 (27/45) vs. 40\% (23/57)] was statistically significant $(\mathrm{P}<0.01)$. The PTEN gene expression between the metastasis and no lymph node metastasis groups [26 (10/38) vs. $63 \%$ $(40 / 64)]$ was significantly different $(\mathrm{P}<0.01)$. The PTEN gene promoter methylation and its protein expression had a significant correlation $(\mathrm{P}=0.042)$. These results suggest that hypermethylation can inactivate the transcription of PTEN and
\end{abstract}

Correspondence to: Professor Yongping Liu, Clinical Oncology Laboratory, Changzhou Tumor Hospital Affiliated to Suzhou University, 1 North Huaide Road, Changzhou, Jiangsu 213002, P.R. China

E-mail: qiyandaisy@126.com

Key words: phosphatase and tensin homologue gene, cervical cancer, mutation, methylation, immunohistochemistry reduce its protein expression. Downregulated PTEN expression is involved in the pathogenesis, invasion and metastasis of cervical cancer, possibly by regulating the balance between apoptosis and proliferation. Therefore, the PTEN expression may be a good marker for the prognosis of cervical cancer.

\section{Introduction}

Cervical cancer is the second most common type of cancer in females worldwide and is a leading cause of cancer-related mortality in females in developing countries. Despite the public health importance of cervical cancer in China, cervical cancer is most common among females, followed by breast cancer, with an estimated 1,520,000 new cases and 780,000 fatalities (1). Multiple epidemiological studies have indicated that the human papillomavirus infection is frequently detected in invasive cervical cancer (2). However, this alone is not sufficient for malignant transformation and other genetic events are required for the development of cancer. The genetic basis underlying cervical tumorigenesis and progression are largely unknown. Recently, much progress has been made in identifying the genes that are involved in the development of cervical cancer, which is useful for understanding the pathogenesis of cervical cancer and defining its molecular signature (3-6).

Phosphatase and tensin homologue (PTEN) is a tumor suppressor gene that is located on human chromosome band 10q23.31, contains 9 exons and encodes a 403 amino acid sequence for a dual-specificity phosphatase with homology to the cytoskeleton proteins, chicken tensin and bovine auxilin, which interact with adhesion molecules (7-10). The mutations of the PTEN gene have been found in a variety of human cancer cell lines and primary tumors of the brain, stomach, breast and kidney in different frequencies (11-15), indicating that the gene may play a role in the tumorigenesis of certain cancers. PTEN promoter methylation and reduced PTEN expression were also found in gastric, non-small cell lung and cervical cancers (16-18). However, little is known regarding the association between mutation, promoter methylation and the PTEN gene expression in cervical cancer in the Chinese population. Therefore, the aim of the present study was to investigate the function of PTEN in cervical cancer and to correlate it further with clinicopathological characteristics to determine its clinical importance. 
Table I. Oligonucleotide primers for phosphatase and tensin homologue analysis.

\begin{tabular}{lllcc}
\hline Exon & \multicolumn{1}{c}{$\begin{array}{c}\text { Forward } \\
\left(5^{\prime}-3^{\prime}\right)\end{array}$} & \multicolumn{1}{c}{$\begin{array}{c}\text { Reverse } \\
\left(5^{\prime}-3^{\prime}\right)\end{array}$} & $\begin{array}{c}\text { Annealing temp. } \\
\left({ }^{\circ} \mathrm{C}\right)\end{array}$ & $\begin{array}{c}\text { Product size } \\
(\mathrm{bp})\end{array}$ \\
\hline 1 & TCCTCCTTTTCTTCAGCCAC & GAAAGGTAAAGAGGAGCAGCC & 56 & 147 \\
2 & TGCATATTTCAGATATTTCTTTCTT & TTTGAAATAGAAAATCAAAGCATTC & 57 & 155 \\
3 & TGTTAATGGTGGCTTTTTG & GCAAGCATACAAATAAGAAAAC & 56 & 114 \\
4 & TTCCTAAGTGCAAAAGATAAC & TACAGTCTATCGGGTTTAAGT & 56 & 147 \\
5 & TTTTTTTTTTATTCTGAGGTTATC & GAAGAGGAAAGGAAAAACATC & 51 & 312 \\
6 & AGTGAAATAACTATAATGGAACA & GAAGGATGAGAATTTCAAGC & 54 & 231 \\
7 & ATCGTTTTTACAGTTTG & TCCCAATGAAAGTAAAGTAGA & 55 & 262 \\
8 & AGGTGACAGATTTTCTTTTTA & TAGCTGTACTCCTAGAATTA & 52 & 394 \\
9 & GTTCATCTGCAAAATGGA & TGGTAATCTGACACAATGTCCTA & 50 & 397 \\
\hline
\end{tabular}

bp, base pair.

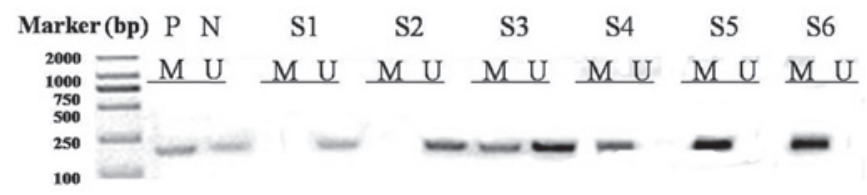

Figure 1. Methylation-specific-polymerase chain reaction (PCR) of phosphatase and tensin homologue (PTEN) in cervical cancer. P, positive control $\mathrm{N}$, unmethylated control; M, methylated PCR products; U, unmethylated PCR products; S1-S6, samples 1 to 6 of the cervical cancer PCR products by methylated primers.

\section{Materials and methods}

Tissue samples. Between January 2011 and April 2012, 102 paraffin blocks of consecutive patients with cervical cancer who underwent a cervical biopsy at the Department of Surgery of Suzhou University, Affiliated Changzhou Tumor Hospital (Jiangsu, China) were enrolled in the study. None of the patients received preoperative chemotherapy and/or radiation therapy. The patients whose surgical tissue was studied provided their written informed consent. Histological studies were also performed and all the specimens were characterized as cervical cancer. The Bioethics Committee of the Institution approved the study. The histological types and grades of tumor were classified according to World Health Organization criteria. The clinical staging of each tumor was established according to the International Federation of Gynecology and Obstetrics (FIGO) criteria.

DNA extraction. Formalin-fixed paraffin samples were cut into $10-\mu \mathrm{m}$ sections. The sections were pulverized under liquid nitrogen using a Mikro-Dismembrator (B. Braun Biotech International, Melsungen, Germany). For each sample, $0.1 \mathrm{~g}$ of pulverized tissue powder was resuspended in $1 \mathrm{ml}$ of $0.25 \%$ xylene and was maintained for $15 \mathrm{~min}$ at $55^{\circ} \mathrm{C}$. The suspension was centrifuged at $14,000 \mathrm{x}$ g for $5 \mathrm{~min}$ and the pellet was suspended in $0.1 \mathrm{ml}$ of xylene and processed as aforementioned for a second time. The resulted sediment was mixed with $100 \%$ ethanol and processed with xylene lysis buffer [(Tris, sodium dodecyl sulfate and ethylenediaminetetraacetic acid (EDTA)].
A lysis buffer containing $300 \mu \mathrm{g} / \mathrm{ml}$ of proteinase $\mathrm{K}$ was added to the pellet, mixed and incubated at $55^{\circ} \mathrm{C}$ overnight. The DNA was extracted by the phenol-chloroform procedure and subsequently dissolved in TE buffer (Tris- $\mathrm{HCl}$ and EDTA) and stored at $-20^{\circ} \mathrm{C}$.

Polymerase chain reaction-single-strand conformational polymorphism (PCR-SSCP). Tumor DNA was subjected to PCR amplification. All 9 exons of the PTEN gene were amplified using primers designed and synthesized by Invitrogen Corp. (Shanghai, China) (Table I). PCR amplification was performed within a reaction mixture containing $2.5 \mu \mathrm{l}$ of 10X PCR buffer $\left(\mathrm{Mg}^{2+}\right.$-free; Takara Bio, Inc., Shiga, Japan), $0.8 \mu \mathrm{Mg}^{2+}(50 \mathrm{mM})$, $0.5 \mu \mathrm{ldNTP}$ (10 mM, Takara), $0.5 \mu \mathrm{l}$ primer F (10 $\mu \mathrm{m}), 0.5 \mu \mathrm{l}$ primer $\mathrm{R}(10 \mu \mathrm{m}), 0.2 \mu \mathrm{l}$ Taq polymerase (5 U/ $\mu \mathrm{l}$, Takara Bio,

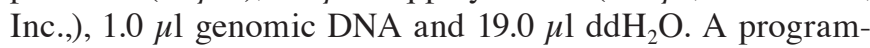
mable thermocycler (Eppendorf PCR system 9700, Hamburg, Germany) was used to perform the amplifications. The PCR condition for each exon was as follows: Pre-denaturation for $5 \mathrm{~min}$ at $95^{\circ} \mathrm{C}, 35$ cycles of $30 \mathrm{sec}$ denaturation at $95^{\circ} \mathrm{C}, 40 \mathrm{sec}$ annealing at the temperature shown in Table I, $60 \mathrm{sec}$ extension at $72^{\circ} \mathrm{C}$ and $10 \mathrm{~min}$ final extension at $72^{\circ} \mathrm{C}$. The amplified PCR products were denatured at $95^{\circ} \mathrm{C}$ for $5 \mathrm{~min}$ and run on an $8 \%$ denaturation polyacrylamide gel (1X Tris/Borate/EDTA buffer, circulatory water) at a voltage of $100 \mathrm{~V}$ and at $10^{\circ} \mathrm{C}$ for $16 \mathrm{~h}$. Silver staining was performed as previously described (19). According to the PCR-SSCP results of the genome DNA, the difference in the single-strand strip number and electrophoresis transference location, also known as the mobility shift, was considered as PCR-SSCP positive. The genome DNA from the positive-PCR-SSCP samples was amplified for bidirectional DNA sequencing. The amplified PCR products were sequenced with an ABI Prism 310 Dye Terminator Cycle Sequencing ready reaction kit (Applied Biosystems, Foster City, CA, USA).

Promoter methylation analysis. Promoter methylation of the PTEN gene was determined by methylation-specific-PCR (MS-PCR). The genomic DNA from carcinoma specimens was subjected to bisulfite modifications using the EZ DNA Methylation-Gold ${ }^{\text {TM }}$ kit (Zymo Research, Irvine, CA, USA) according to the manufacturer's instructions. The modified 

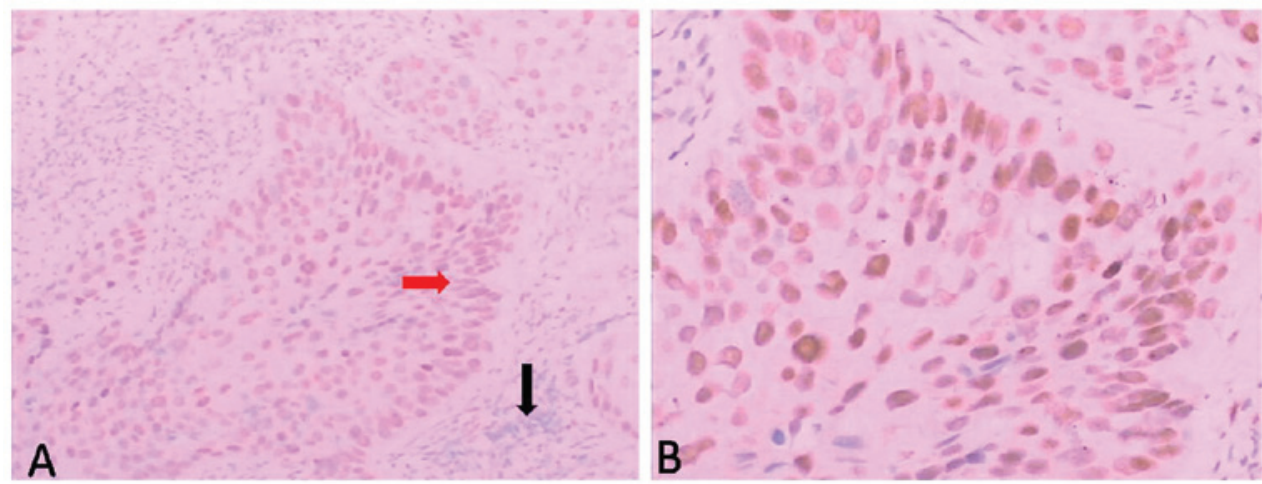

Figure 2. Immunohistochemical staining of phosphatase and tensin homologue (PTEN) protein in cervical cancer. (A) The black arrowhead shows the PTEN expression and the red arrowhead shows the loss of staining, indicating a loss in PTEN expression (original magnification, x100). (B) Amplification of red arrowhead area (magnification, $\mathrm{x} 200$ ).

DNA was used as a template for MS-PCR as described above. The primers for the unmethylated PTEN were forward, 5'-TTTTTTGGGTTTTTGAAATTTAATG-3' and reverse, 5'-AACCTACTATTATATCACCAACATA-3' yielding a 165-bp product. The primers for the methylated PTEN were forward, 5'-GTTTTTTGGGTTTTTGAAATT TAAC-3' and reverse, 5'-AACCTACTATTATATCGCCAA CGTA-3', yielding a 166-bp product (Fig. 1). The $25 \mu \mathrm{l}$ of PCR mixture contained $10 \mathrm{mM}$ Tris- $\mathrm{HCl}$ (pH 8.3), $50 \mathrm{mM} \mathrm{KCl,} 1.25 \mathrm{mM}$ $\mathrm{MgCl}_{2}, 100 \mu \mathrm{M}$ dNTP, $0.6 \mu \mathrm{M}$ of each primer, 1 unit of Taq DNA polymerase (HotStarTaq; Takara Bio, Inc.) and bisulfite modified DNA (25 ng) (20). The PCR conditions consisted of a denaturing step at $95^{\circ} \mathrm{C}$ for 5 min and 35 cycles of $94^{\circ} \mathrm{C}$ for $30 \mathrm{sec}, 60^{\circ} \mathrm{C}$ (for the detection of the unmethylated PTEN gene) or $62^{\circ} \mathrm{C}$ (for the detection of the methylated PTEN gene) for $30 \mathrm{sec}$ and $72^{\circ} \mathrm{C}$ for $60 \mathrm{sec}$, followed by a final extension at $72^{\circ} \mathrm{C}$ for $10 \mathrm{~min}$. Each PCR product $(20 \mu \mathrm{l})$ was directly loaded onto a $2 \%$ agarose gel, stained with ethidium bromide and visualized under UV illumination. The DNA from the GenoMethtm Universal Methylated DNA Standard (Zymo Research, Irvine, CA, USA)was used as a positive control for the methylated alleles. Water and unmodified DNA were used as negative controls for every batch reaction. The PCR for all the samples that demonstrated methylation for the individual genes was repeated to confirm these results.

Immunohistochemical analysis. Phosphate-buffered saline (PBS) was used instead of the primary antibody for the blank control and normal non-immunized rabbit serum was used instead of the primary antibody for the negative control. Following the manufacturer's instructions provided with the immunohistochemistry staining reagent kit (Boster, Wuhan, China), the deparaffinized tissues were cut into $5-\mu \mathrm{m}$ thick sections, washed 3 times ( 5 min each time) with PBS, incubated at room temperature in $3 \% \mathrm{H}_{2} \mathrm{O}_{2}$ to eliminate the endogenous peroxidase activity and subsequently washed an additional 3 times ( 3 min each time) with PBS. The antigens were prepared in a microwave (citrate buffer, $\mathrm{pH} 6.0$ ), naturally refrigerated to room temperature, washed 3 times ( 3 min each time) with PBS, incubated at room temperature with normal non-immunized serum solution for $15 \mathrm{~min}$ to indicate the non-specific sites, incubated at room temperature with the primary antibody solution and horseradish peroxidase-tagged streptavidin for $15 \mathrm{~min}$

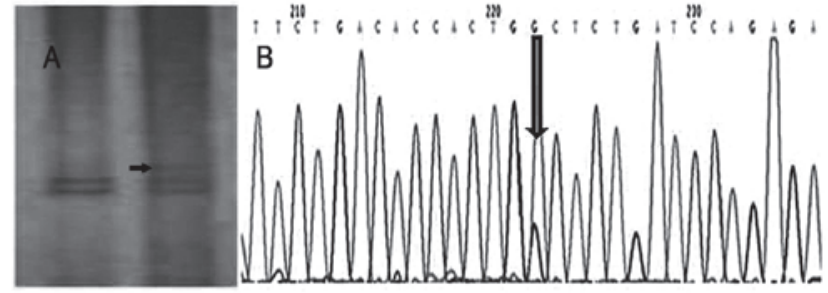

Figure 3. (A) Polymerase chain reaction-single-stranded conformational polymorphism showing the abrupt band patterns of exon 9 of the phosphatase and tensin homologue (PTEN) gene (case no. 36). (B) DNA sequence analysis of the PTEN gene in a representative sample (case no. 36) with a mutation in exon 9 showing a A to $\mathrm{G}$ point mutation.

and finally washed 3 times (3 min each time) with PBS. The Drosophila Anion Exchanger (DAE) stain was rinsed with PBS for $3 \mathrm{~min}$, counterstained with hematoxylin for $1 \mathrm{~min}$, rinsed with tap water for 2 min, differentiated with $1 \%$ hydrochloric ethanol, rinsed with tap water for $5 \mathrm{~min}$, dehydrated with gradient alcohol and transparentized with dimethylbenzene. The sections were coated with neutral balata. PTEN-positive stained slides from patients with cervicitis were used as the positive control in each set of experiments. For the negative control, the same procedure was followed with the exception of the primary antibody. The staining was graded as either negative (-) or positive $(+)$. The specimens in which $\geq 10 \%$ of the neoplastic cell cytosols showed a positive immunoreactivity were considered to be immunoreactive (Fig. 2). The specificity of the PTEN antibody (OriGene, Rockville, MD, USA) is an immunoglobulin G1 mouse monoclonal antibody against human PTEN.

Statistical analysis. The $\chi^{2}$ test was used to determine the correlation of the promoter methylation and the PTEN gene expression with the clinicopathological parameters. The association between the promoter methylation with the PTEN gene expression was also analyzed using the $\chi^{2}$ test. $\mathrm{P}<0.05$ was considered to indicate a statistically significant difference.

\section{Results}

Mutational analysis. A total of 102 cases of cervical cancer were studied. The PTEN novel mutations were identified in 
Table II. Association between promoter methylation of the PTEN gene with the clinicopathological features in cervical cancer patients

\begin{tabular}{|c|c|c|c|}
\hline Characteristics & No. of cases tested $(n=102)$ & Methylation of PTEN present (\%) & P-value \\
\hline \multicolumn{4}{|l|}{ Age, years } \\
\hline$\leq 44$ & 44 & $27 / 44(61)$ & \multirow[t]{2}{*}{0.552} \\
\hline$>44$ & 58 & $36 / 58(62)$ & \\
\hline \multicolumn{4}{|l|}{ FIGO stage } \\
\hline I-II & 45 & $23 / 45(51)$ & \multirow[t]{2}{*}{0.039} \\
\hline III-IV & 57 & $40 / 57(70)$ & \\
\hline \multicolumn{4}{|l|}{ Tumor grade } \\
\hline G1 & 58 & $30 / 58(52)$ & \multirow[t]{2}{*}{0.014} \\
\hline G2-G3 & 44 & $33 / 44(75)$ & \\
\hline \multicolumn{4}{|l|}{ Tumor size, cm } \\
\hline$<4$ & 63 & $34 / 63(54)$ & \multirow[t]{2}{*}{0.031} \\
\hline$\geq 4$ & 39 & $29 / 39(74)$ & \\
\hline \multicolumn{4}{|l|}{ Histology type } \\
\hline $\mathrm{SCC}$ & 78 & $47 / 78(60)$ & \multirow[t]{2}{*}{0.376} \\
\hline $\mathrm{AD}$ & 24 & $16 / 24(67)$ & \\
\hline \multicolumn{4}{|c|}{ Lymph node metastasis } \\
\hline With & 38 & $32 / 38(84)$ & \multirow[t]{2}{*}{0.001} \\
\hline Without & 64 & $31 / 64(48)$ & \\
\hline
\end{tabular}

PTEN, phosphatase and tensin homologue; FIGO, International Federation of Gynecology and Obstetrics; SCC, squamous-cell carcinoma; $\mathrm{AD}$, adenocarcinoma.

one of $102(1 \%)$ tumor specimens. The SSCP analysis showed an abnormal band on exon 9 of the PTEN gene (Fig. 3A). Direct sequencing analysis showed the mutation site in exon 9 was found at codon 380 (CAC-GGC), resulting in an aspartic acid to glycine substitution (Fig. 3B). No mutation was found in exons 1 to 8 . The clinical parameters of the mutated patient were evaluated. This patient was 63 years old and was found to be present in clinical stage III and pathological grade 2 .

Promoter methylation of the PTEN gene. The PTEN promoter methylation was analyzed in tumor samples from 102 cervical cancer patients. Among the tested cases, the PTEN promoter methylation was detected in 63 of $102(62 \%)$ cases in the tumor DNA. Table II shows the association between methylation and the clinicopathological parameters. Among the patients examined for PTEN methylation, 51\% (23 of 45) of cases were methylated in clinical stage I-II and 70\% (40 of 57) in stages III-IV. The correlation between PTEN methylation and clinical stages was found to be statistically significant $(\mathrm{P}=0.039)$. Furthermore, the study showed that PTEN methylation was not significantly correlated with the histology type and age of the patients, but was significantly correlated with the carcinoma size, lymph node metastasis and tumor grade $(\mathrm{P}<0.05)$.

PTEN protein expression in patients with cervical cancer. The immunohistochemical analysis of the PTEN gene was performed in 102 cases of cervical carcinoma and 15 samples of cervicitis (inflammatory lesion) as a control. Nuclear PTEN expression was detected in all the 15 samples of cervicitis and $63 / 102(62 \%)$ cases of cervical cancer (Table III). A decreased
PTEN expression was observed with the increase in clinical stage (40 and 59\% in stages I-II and III-IV, respectively) and a statistically significant correlation was also observed between the loss of PTEN expression and lymph node metastasis. The lymph node metastasis group showed more expressional loss when compared to the specimens belonging to the without lymph node metastasis group $(\mathrm{P}=0.001)$.

Correlation of PTEN expression with promoter methylation. The correlation of the promoter methylation with the PTEN gene expression was determined in 102 cases of cervical cancer. Among 102 cases, 63 were found to be methylated, whereas 37 were identified as unmethylated. The loss of PTEN expression was observed in 24 of the 63 methylation-positive cases, whereas only 15 of the 39 methylation-negative cases were observed as having a loss of PTEN expression. The association was found to be statistically significant, $\mathrm{P}=0.042$ (Table IV).

\section{Discussion}

PTEN, a tumor-suppressing gene, is involved in cellular differentiation, reproduction and apoptosis, as well as cellular adhesion and mobility. The loss or downregulation of PTEN plays an important role in the multiple steps of tumorigenesis and progression of malignancies, and mutations and deletions of this gene have been described in a wide range of human cancers. The incidence is $30-50 \%$ in endometrial cancers, $25 \%$ in glioblastomas, $21 \%$ in ovarian cancer, $13 \%$ in prostate cancer and $<5 \%$ in breast and thyroid cancer $(11,19-21)$. The mutation of PTEN in cervical carcinoma does not appear to be 
Table III. Association between the expression of the PTEN protein and the clinicopathological features in cervical cancer.

\begin{tabular}{lcc}
\hline Characteristics & No. of cases tested $(\mathrm{n}=102)$ & Presence of PTEN expression $(\%)$ \\
\hline Age, years & & $22 / 44(50)$ \\
$\quad \leq 44$ & 44 & $28 / 58(48)$ \\
$>44$ & 58 & $27 / 45(60)$ \\
FIGO stage & & $23 / 57(40)$ \\
I-II & 45 & \\
III-IV & 57 & $32 / 58(55)$ \\
Tumor grade & & $18 / 44(41)$ \\
G1 & 58 & $34 / 63(54)$ \\
G2-G3 & 44 & $16 / 39(41)$ \\
Tumor size, cm & & \\
$<4$ & 63 & $38 / 78(49)$ \\
$\geq 4$ & 39 & $12 / 24(50)$ \\
Histology type & & \\
SCC & 78 & $10 / 38(26)$ \\
AD & 24 & $40 / 64(63)$ \\
Lymph node metastasis & & 0.110 \\
With & 38 & 0.143 \\
Without & 64 & 0.549 \\
\hline
\end{tabular}

PTEN, phosphatase and tensin homologue; FIGO, International Federation of Gynecology and Obstetrics; SCC, squamous-cell carcinoma; $\mathrm{AD}$, adenocarcinoma.

frequent. Yaginuma et al (22) reported that one in 43 cervical cancers $(2 \%)$ had a PTEN mutation in a Japanese population. Rizvi et al (23) reported that three of 135 cervical cancers (2\%) had a PTEN mutation in an Indian population. However, in a Chinese population, Cheung et al (24) reported 0 of 60 cervical cancers had a PTEN mutation. In the present study, entire coding regions of the PTEN gene were screened for the mutation by PCR-SSCP in 102 cases of cervical cancer. The results showed an A to $G$ point mutation on exon 9 of the PTEN gene in one case only (case number 36 ), and this mutation lead to a missense mutation on codon 380, which caused a transition of aspartic acid into glycine. The exact function of this mutation is still unknown, and functional analysis is required to investigate this. The rate of the PTEN gene mutation is $1 \%$ in the patients with cervical cancer from a Chinese population. It was also reported that PTEN expression was frequently diminished at the transcriptional level. DNA methylation, transcriptional repression and microRNA-directed mRNA degradation and translational disruption appear to reduce PTEN expression in certain cancers $(25,26)$. Therefore, it may be concluded that the PTEN mutation is not common in Chinese cervical cancer.

Recent studies have also shown that the $\mathrm{CpG}$ islands of the PTEN promoter are methylated in various human malignancies including cervical cancer, but the PTEN methylation data were different. In a study by Yang et al (18), PTEN methylation was observed in 20 of 127 (16\%) cervical cancers, whereas it was reported in 36 of $62(58 \%)$ squamous cell cervical cancers in the study by Cheung et al (24). There was no PTEN expression observed in three of 10 and 0 of 10 PTEN methylation-negative and -positive cases, respectively. The preliminary data from the study by Eijsink et al (27) showed
Table IV. Correlation of promoter methylation with PTEN expression in cervical cancer.

\begin{tabular}{lccc}
\hline & \multicolumn{2}{c}{ PTEN expression } & \\
\cline { 2 - 3 } $\begin{array}{l}\text { PTEN promoter } \\
\text { methylation }\end{array}$ & Positive & Negative & P-value \\
\hline Positive & 26 & 37 & 0.042 \\
Negative & 24 & 15 & \\
\hline
\end{tabular}

PTEN, phosphatase and tensin homologue.

the presence of PTEN gene promoter methylation in four of $19(21 \%)$ cases. No PTEN immunostaining was observed in these four PTEN methylation-positive cases. Rizvi et al (23) reported that in the PTEN gene, there was methylation in $61 \%$ (83/135) of specimens and a loss of PTEN expression in $41 \%$ (34/83) of methylation-positive cases, whereas in the 52 methylation-negative cases only $25 \%(13 / 52)$ were observed as immunostaining-negative, which was statistically significant $(\mathrm{P}=0.05)$. In the present study, PTEN promoter methylation was detected in 63 of $102(62 \%)$ cases in the tumor DNA. PTEN methylation was significantly correlated with the FIGO stage, carcinoma size, lymph node metastasis and tumor grade $(\mathrm{P}<0.05)$. Any discrepancies between the data of the PTEN methylation from the high and the low rates may be due to the number of evaluated patients and/or the samples with/without the radiotherapy and/or chemotherapy. The patients in the study by Yang et al (18) received radiotherapy 
and/or chemotherapy, and the study by Eijsink et al (27) only analyzed 19 cases.

Furthermore, the present study showed that the PTEN protein expression in the 102 cervical cancer tissue samples was significantly correlated with the FIGO stage and lymph node metastasis $(\mathrm{P}<0.05)$. The Lu et al $(28)$ study showed that the level of PTEN expression in the metastatic pelvic lymph node group was significantly lower compared to the non-metastatic group $(\mathrm{P}<0.01)$. The Eijsink et al $(27)$ study indicated that a loss of PTEN expression frequently occurs in early-stage cervical cancer and this is associated with pelvic lymph node metastasis $(\mathrm{P}=0.019)$, but not to the survival rate. These experimental results suggest that there is a role for the loss of PTEN in determining the metastatic potential of tumors.

Therefore, a correlation between the occurrence of PTEN promoter methylation with its reduced expression was also formulated. Promoter methylation of PTEN was significantly correlated with the loss of PTEN expression and showed a significant association $(\mathrm{P}=0.042)$. These results indicate that a reduced PTEN expression may be due to PTEN promoter methylation. Promoter methylation of PTEN presents a major alternative mechanism of gene silencing.

In conclusion, abnormal promoter methylation of the PTEN gene is usually found in cervical cancer and is associated with tumor differentiation, infiltrating depth, lymph node metastasis and FIGO staging. PTEN may play an important role in the occurrence and development of cervical cancer, and the PTEN protein expression phenotype can be considered as an indicator for the pathophysiological behavior of cervical cancer.

\section{Acknowledgements}

The authors would like to thank the personnel at the Changzhou Tumor Hospital (Changzhou, China) who were involved in the present study. The study was partly supported by the Natural Science Foundation of China (grant nos. 81071799 and 81372212), the Natural Science Foundation of Jiangsu (grant nos. BK2011251 and BL2013012), the Health Talents Project for Jiangsu, China (grant nos. LJ201157, RC2011038 and BRA2011038), the Research of Health Department in Jiangsu (grant no. Z201221), the Talents Project ('831' and health) of Changzhou Municipality, the Science and Technology Planning Project of Changzhou Health Bureau, Jiangsu (grant nos. QN201106 and ZD201203), the 333 Talents Training Project of Jiangsu Province, the Key Medical Innovation Talents Training Project of Changzhou, Jiangsu, and the Project of Jiangsu Province Sanitation Innovation Team.

\section{References}

1. Ferlay J, Shin HR, Bray F, et al: Estimates of worldwide burden of cancer in 2008: GLOBOCAN 2008. Int J Cancer 127: 2893-2917, 2010.

2. Walboomers JM, Jacobs MV, Manos MM, et al: Human papillomavirus is a necessary cause of invasive cervical cancer worldwide. J Pathol 189: 12-19, 1999.

3. Noordhuis MG, Eijsink JJ, Ten HK, et al: Expression of epidermal growth factor receptor (EGFR) and activated EGFR predict poor response to (chemo) radiation and survival in cervical cancer. Clin Cancer Res 15: 7389-7397, 2009.
4. Woelber L, Kress K, Kersten JF, et al: Carbonic anhydrase IX in tumor tissue and sera of patients with primary cervical cancer. BMC Cancer 11: 12, 2011.

5. Hellberg D, Tot T and Stendahl U: Pitfalls in immunohistochemical validation of tumor marker expression - exemplified in invasive cancer of the uterine cervix. Gynecol Oncol 112: 235-240, 2009.

6. Khunamornpong S, Settakorn J, Sukpan K, et al: Cyclooxygenase-2 expression in squamous cell carcinoma of the uterine cervix is associated with lymph node metastasis. Gynecol Oncol 112: 241-247, 2009.

7. Lima EM, Araújo JJ, Harada ML, et al: Molecular study of the tumour suppressor gene PTEN in gastric adenocarcinoma in Brazil. Clin Exp Med 5: 129-132, 2005.

8. Oki E, Kakeji Y, Baba H, et al: Impact of loss of heterozygosity of encoding phosphate and tensin homolog on the prognosis of gastric cancer. J Gastroenterol Hepatol 21: 814-818, 2006.

9. Stiles BL: Phosphatase and tensin homologue deleted on chromosome 10: extending its PTENtacles. Int J Biochem Cell Biol 41: 757-761, 2009.

10. Raftopoulou M, Etienne-Manneville S, Self A, et al: Regulation of cell migration by the $\mathrm{C} 2$ domain of the tumor suppressor PTEN. Science 303: 1179-1181, 2004.

11. Li J, Yen C, Liaw D, et al: PTEN, a putative protein tyrosine phosphatase gene mutated in human brain, breast and prostate cancer. Science 275: 1943-1947, 1997.

12. Guo CY, Xu XF, Wu JY and Liu SF: PCR-SSCP-DNA sequencing method in detecting PTEN gene mutation and its significance in human gastric cancer. World J Gastroenterol 14: 3804-3811, 2008.

13. Marsit CJ, Zheng S, Aldape K, et al: PTEN expression in non-small-cell lung cancer: evaluating its relation to tumor characteristics, allelic loss, and epigenetic alteration. Hum Pathol 36: 768-776, 2005.

14. Teng Y, Sun AN, Pan XC, et al: Synergistic function of Smad4 and PTEN in suppressing forestomach squamous cell carcinoma in the mouse. Cancer Res 66: 6972-6981, 2006.

15. Perrone F, Lampis A, Orsenigo M, et al: PI3KCA/PTEN deregulation contributes to impaired responses to cetuximab in metastatic colorectal cancer patients. Ann Oncol 20: 84-90, 2009.

16. Kang YH, Lee HS and Kim WH: Promoter methylation and silencing of PTEN in gastric carcinoma. Lab Invest 82: 285-291, 2002.

17. Soria JC, Lee HY, Lee JI, et al: Lack of PTEN expression in non-small cell lung cancer could be related to promoter methylation. Clin Cancer Res 8: 1178-1184, 2002.

18. Yang HJ, Liu VW, Wang Y, et al: Differential DNA methylation profiles in gynecological cancers and correlation with clinico-pathological data. BMC Cancer 6: 212, 2006.

19. Jiang YA, Fan LF, Jiang CQ, et al: Expression and significance of PTEN, hypoxia-inducible factor-1 alpha in colorectal adenoma and adenocarcinoma. World J Gastroenterol 9: 491-494, 2003.

20. Schöndorf T, Ebert MP, Hoffmann J, et al: Hypermethylation of the PTEN gene in ovarian cancer cell lines. Cancer Lett 207: 215-220, 2004

21. Steck PA, Pershouse MA, Jasser SA, et al: Identification of a candidate tumour suppressor gene, MMAC1, at chromosome 10 q23.3 that is mutated in multiple advanced cancers. Nat Genet 15: 356-362, 1997.

22. Yaginuma $\mathrm{Y}$, Yamashita $\mathrm{T}$, Ishiya $\mathrm{T}$, et al: Abnormal structure and expression of PTEN/MMAC1 gene in human uterine cancers. Mol Carcinog 27: 110-116, 2000.

23. Rizvi MM, Alam MS, Ali A, et al: Aberrant promoter methylation and inactivation of PTEN gene in cervical carcinoma from Indian population. J Cancer Res Clin Oncol 137: 1255-1262, 2011.

24. Cheung TH, Lo KW, Yim SF, et al: Epigenetic and genetic alternation of PTEN in cervical neoplasm. Gynecol Oncol 93: 621-627, 2004.

25. Wiencke JK, Zheng S, Jelluma N, et al: Methylation of the PTEN promoter defines low-grade gliomas and secondary glioblastoma. Neuro Oncol 9: 271-279, 2007.

26. Yang $\mathrm{H}$, Kong $\mathrm{W}, \mathrm{He} \mathrm{L}$, et al: MicroRNA expression profiling in human ovarian cancer: miR-214 induces cell survival and cisplatin resistance by targeting PTEN. Cancer Res 68: 425-433, 2008.

27. Eijsink JJ, Noordhuis MG, ten Hoor KA, et al: The epidermal growth factor receptor pathway in relation to pelvic lymph node metastasis and survival in early-stage cervical cancer. Hum Pathol 41: 1735-1741,2010.

28. Lu D, Qian J, Yin X, et al: Expression of PTEN and survivin in cervical cancer: promising biological markers for early diagnosis and prognostic evaluation. Br J Biomed Sci 69: 143-146, 2012. 